\title{
Responsabilité sociale des facultés de médecine et accréditation
}

\author{
Social accountability of medicals schools and accreditation
}

\author{
Geneviève MOINEAU \\ 1 Présidente-directrice générale de l'Association des facultés de médecine du Canada
}

Manuscrit reçu le 17 novembre 2015 ; commentaires éditoriaux formulés à l'auteure le 7 janvier 2016 ; accepté pour publication le 20 janvier 2016

\begin{abstract}
Mots-clés
Responsabilité

sociale ;

Accréditation

\section{Keywords}

Social accountability;

Medical schools;

North America;

Accreditation

Résumé - Contexte et problématique : Les facultés de médecine de la francophonie sont de plus en plus axées sur l'importance de définir leur mandat en matière de responsabilité sociale et d'harmonier leur mission et leurs objectifs en conséquence. Parallèlement, l'Educational Commission for Foreign Medical Graduates (ECFMG) exige que les diplômés qui désirent poursuivre leur formation aux États-Unis au-delà de 2023 proviennent de facultés de médecine agréées/accréditées. Or, la plupart des facultés francophones ne le sont pas. Objectifs et exégèse : Le présent document décrit les efforts déployés par le Canada pour définir clairement le mandat des facultés de médecine en matière de responsabilité sociale et la manière dont cet élément clé de la mission en est venu à être incorporé aux normes d'accréditation du Comité d'agrément des facultés de médecine du Canada (CAFMC). En plus d'accorder l'accréditation aux facultés de médecine canadiennes, le CAFMC est pleinement reconnu par la Fédération mondiale pour l'éducation médicale. Conclusion : Le Canada se réjouit à l'idée de poursuivre son partenariat avec les facultés de médecine francophones du monde entier pour contribuer à l'avancement de la responsabilité sociale et de l'accréditation.

Abstract - Context and issues: Faculties of medicine within the French-speaking world are increasingly focused on the importance of defining their social accountability and aligning their mission and goals accordingly. Concurrently, the ECFMG is requiring that graduates who want to train in the US after 2023 come from accredited medical schools. However, most French-speaking schools are not accredited. Objectives and analysis: This paper describes Canada's efforts to clearly define the social-accountability mandate of medical schools and how this key concept in their mission has now been incorporated in the accreditation standards of the Committee on Accreditation of Canadian Medical Schools (CACMS). CACMs not only accredits Canadian medical schools but has received full recognition by the World Federation for Medical Education. Conclusion: Canada looks forward to continuing to partner with French-speaking schools around the world to advance social accountability and accreditation.
\end{abstract}




\section{Introduction et problématique}

Les facultés de médecine de l'ensemble de la francophonie placent aujourd'hui plus que jamais la responsabilité sociale au rang de leurs priorités. L'un des conseils de la Conférence internationale des doyens et des facultés de médecine d'expression française porte en effet sur ce thème.

À compter de 2023, les États-Unis prévoient n'autoriser à exercer que des médecins diplômés de facultés accréditées par un organisme reconnu sur le plan international. Dans cette optique, il est du devoir des facultés de médecine de se pencher sans tarder sur cette problématique.

Les deux concepts sont-ils mutuellement exclusifs ? Il semble que non. Les 17 facultés de médecine canadiennes sont accréditées par le Comité sur l'agrément des facultés de médecine du Canada (CAFMC) et par le Liaison Committee on Medical Education (LCME) des États-Unis. Ces deux organismes sont tous deux reconnus par la Fédération mondiale pour l'éducation médicale (World Federation for Medical Education -WFME). Nos facultés ont également accepté d'être régies par une norme d'accréditation/ agrément très concrète en ce qui a trait à la responsabilité sociale.

\section{Contexte}

L'Association des facultés de médecine du Canada (AFMC) est constituée du partenariat des 17 facultés de médecine canadiennes. Les doyens des facultés siègent au conseil d'administration de l'AFMC en compagnie de quatre représentants du public. L'AFMC vise l'excellence sur le plan de l'éducation, de la recherche et des soins. Depuis le début du $\mathrm{XXI}^{\mathrm{e}}$ siècle, elle est parvenue à amener les facultés de médecine canadiennes à accorder une importance grandissante au concept de responsabilité sociale. Elle est, de concert avec l'Association médicale canadienne, un organisme de parrainage du CAFMC.

\section{Objectif de la contribution}

En s'appuyant sur l'expérience de l'AFMC, le présent article vise à décrire les recommandations adoptées pour l'ensemble des facultés, tant au niveau prédoctoral que postdoctoral. Ces recommandations confirment l'importance de la responsabilité sociale pour les facultés de médecine canadiennes et exposent la nouvelle norme d'accréditation proposée en matière de responsabilité sociale.

\section{La prise en compte de la responsabilité sociale dans le cadre de l'accréditation des facultés de médecine du Canada}

Des rapports préparatoires et des recommandations

En 2001, en collaboration avec le ministère de la Santé du Canada, l'AFMC publie une réflexion avec l'approbation de tous les doyens des facultés de médecine du pays. Le document a pour titre : Imputabilité sociale: Une vision pour les facultés de médecine du Canada ${ }^{[1]}$.

Cette publication donne le coup d'envoi à la tenue d'une consultation pancanadienne sur l'éducation médicale. Publié en 2010, le premier rapport sur la question s'intitule: L'Avenir de l'éducation médicale au Canada (AEMC) : Une vision collective pour les études médicales prédoctorales ${ }^{[2]}$. Au total, dix secteurs prioritaires sont déterminés à partir des données probantes recueillies dans le cadre du projet sur l'AEMC. Les recommandations élaborées s'articulent autour de ces dix secteurs (voir le Tableau I). Le concept de responsabilité sociale est visiblement présent dans plusieurs de ces recommandations.

La première recommandation, "Répondre aux besoins individuels et communautaires », confirme que la responsabilité sociale et l'imputabilité sont des valeurs fondamentales sur lesquelles reposent les rôles des médecins et des facultés de médecine au Canada. Cet engagement signifie que, sur les plans 
Tableau I. L'Avenir de l'éducation médicale au Canada Volet prédoctoral.

\begin{tabular}{|l|l|}
\hline & Recommandations - Secteurs prioritaires \\
\hline I & Répondre aux besoins individuels et communautaires \\
II & Améliorer les processus d'admission \\
III & Miser sur les assises scientifiques de la médecine \\
IV & Promouvoir la prévention et la santé publique \\
V & Exposer le curriculum caché \\
VI & Diversifier les contextes d'apprentissage \\
VII & Valoriser le généralisme \\
VIII & Faire progresser la pratique interprofessionnelle et intra professionnelle \\
IX & Adopter une approche flexible et fondée sur les compétences \\
X & Favoriser le leadership médical \\
\hline & Recommandations - Recommandations habilitantes \\
\hline A & Rajuster les normes d'agrément \\
B & Développer la capacité de changer \\
C & Accroître la collaboration nationale \\
D & Améliorer l'utilisation de la technologie \\
E & Renforcer le perfectionnement professoral \\
\hline
\end{tabular}

individuel et collectif, les médecins et les facultés doivent répondre aux besoins diversifiés des individus et des collectivités partout au Canada, et s'acquitter des responsabilités internationales envers la communauté mondiale.

La sixième recommandation, «Diversifier les contextes d'apprentissage ", confirme que dans la prestation du continuum des soins médicaux, les médecins canadiens exercent dans une grande diversité de milieux institutionnels et communautaires. Pour les préparer à ces réalités, les facultés de médecine doivent offrir à tous les étudiants des expériences d'apprentissage, durant toutes les études médicales prédoctorales dans divers milieux, allant des petites collectivités rurales jusqu'aux centres hospitaliers de soins tertiaires complexes.

La huitième recommandation insiste sur l'importance de «Faire progresser la pratique interprofessionnelle et intra-professionnelle ». Pour améliorer les soins en collaboration centrés sur le patient, les études médicales prédoctorales doivent tenir compte des changements constants dans les champs de pratique et la prestation des soins de santé. Les facultés de médecine doivent faire en sorte que les apprenants au niveau prédoctoral acquièrent les compétences leur permettant de fonctionner efficacement en tant que membres d'équipes interprofessionnelles et intraprofessionnelles.

La consultation suivante a porté sur la formation des résidents, tant en médecine générale que dans les autres spécialités. Au terme de cette consultation, a été produit le rapport sur le volet postdoctoral de l'Avenir de l'éducation médicale au Canada (AEMC EMPo ${ }^{[3]}$. On constate une fois de plus l'importance de la responsabilité sociale dans les dix recommandations (voir Tableau II).

La première recommandation est au cœur de la responsabilité du milieu médical: "Assurer un mélange judicieux, une répartition appropriée et un nombre suffisant de médecins pour répondre aux besoins de la société ». Dans le contexte d'un système de soins de santé en pleine évolution, le système d'EMPo doit continuellement ajuster ses programmes de formation de manière à favoriser un juste équilibre sur le plan de la diversité, de la répartition et du nombre de généralistes et de spécialistes, notamment en ce qui a trait aux cliniciens-chercheurs, aux enseignants et aux leaders, afin de répondre aux besoins de la population canadienne et de s'acquitter de ses responsabilités envers elle. Travaillant en partenariat avec tous les fournisseurs de soins de santé et les intervenants du domaine, les médecins doivent traiter des 
Tableau II. L'Avenir de l'éducation médicale au Canada - Volet postdoctoral.

\begin{tabular}{|c|c|}
\hline & Recommandations \\
\hline 1 & $\begin{array}{l}\text { Assurer un mélange judicieux, une répartition appropriée et un nombre suffisant de médecins pour répondre } \\
\text { aux besoins de la société }\end{array}$ \\
\hline 2 & $\begin{array}{l}\text { Cultiver la responsabilité sociale par le biais de l'expérience acquise dans divers milieux d'apprentissage et } \\
\text { de travail }\end{array}$ \\
\hline 3 & Créer des milieux positifs propices à l'apprentissage et au travail \\
\hline 4 & Intégrer des programmes de formation fondés sur les compétences dans les programmes postdoctoraux \\
\hline 5 & Assurer une intégration et des transitions efficaces tout au long du continuum pédagogique \\
\hline 6 & Mettre en œuvre des systèmes d'évaluation efficaces \\
\hline 7 & Former, appuyer et reconnaître les enseignants-cliniciens \\
\hline 8 & Encourager le développement du leadership \\
\hline
\end{tabular}

divers besoins des particuliers et des collectivités de l'ensemble du Canada en matière de santé et de mieux-être.

Deux des mesures transformatrices clés consistent à : 1) créer ou recueillir des données probantes permettant d'évaluer les besoins au niveau provincial et en matière de ressources en santé et : 2) à créer une approche nationale, fondée sur des données solides, afin d'établir et d'ajuster, selon les besoins, le nombre de postes de résidence requis dans les différentes spécialités de manière à répondre aux besoins sociétaux.

Dans la deuxième recommandation, «Cultiver la responsabilité sociale par le biais de l'expérience acquise dans divers milieux d'apprentissage et de travail », nous avons confirmé que répondre aux divers besoins des Canadiens, actuels et émergents, en matière de soins de santé implique un engagement à la fois individuel et collectif envers l'imputabilité sociale. Les programmes d'EMPo devraient procurer une expérience d'apprentissage et de travail dans divers milieux afin de cultiver l'imputabilité sociale chez les résidents et orienter leur choix de pratique future. La mesure transformatrice clé consiste à offrir à tous les résidents des milieux d'apprentissage diversifiés comprenant des milieux d'exercice variés et à les exposer à une vaste gamme de modèles de prestation de services.

En résumé, nous avons élaboré des recommandations qui placent les Canadiens au centre de l'éducation médicale, sont axées sur les patients et favorisent les soins collaboratifs, reconnaissent les besoins communautaires et accordent de la valeur aux soins communautaires, et insistent sur l'importance de faire du généralisme la pierre angulaire de l'ensemble des diplômés en médecine.

\section{Des procédures d'accréditation/agrément actualisées}

Depuis sa création en 1979, le CAFMC était tenu d'utiliser les mêmes normes et procédures que le LCME.

En 2013, l'AFMC, l'Association médicale canadienne (AMC), l'Association of American Medical Colleges (AAMC) et l'American Medical Association (AMA), organismes qui parrainent respectivement le CAFMC et le LCME, ont signé un protocole d'entente marquant une nouvelle ère dans l'accréditation des facultés de médecine au Canada. Cette entente permet désormais au Canada de modifier les éléments des normes et de déterminer ses propres procédures. Les facultés de médecine canadiennes continueront à recevoir l'accréditation concurrente du LCME et les diplômés continueront de bénéficier de la réciprocité avec les États-Unis.

En 2014, le Comité d'agrément des facultés de médecine du Canada (CAFMC) a mis en place ses propres éléments et normes d'accréditation, ainsi que ses règles de procédure, qui sont mieux adaptés aux besoins des Canadiens et entièrement approuvés par le Liaison Committee on Medical Education (LCME).

Cette démarche représente aussi une première étape en vue d' «harmoniser les normes d'agrément » tout au long du continuum de l'éducation médicale, 
Tableau III. Comité d'agrément des facultés de médecine du Canada (CAFMC).

\begin{tabular}{|l|l|}
\hline & Normes d'agrément \\
\hline Norme 1 & Mission, planification, organisation et intégrité \\
Norme 2 & Direction et administration \\
Norme 3 & Milieux universitaire et d'apprentissage \\
Norme 4 & Préparation, productivité et participation des membres du corps professoral et politiques connexes \\
Norme 5 & Ressources pédagogiques et infrastructure \\
Norme 6 & Compétences, objectifs et conception du programme d'études \\
Norme 7 & Contenu du programme d'études \\
Norme 8 & Gestion, évaluation et amélioration du programme d'études \\
Norme 9 & Enseignement, supervision, évaluation et sécurité des étudiants et des patients \\
Norme 10 & Sélection, affectation et progrès des étudiants en médecine \\
Norme 11 & Aide pédagogique et au choix de carrière et dossiers scolaires des étudiants en médecine \\
Norme 12 & Services de santé, de conseils personnalisés et d'aide financière à 1 intention des étudiants en \\
& médecine \\
\hline
\end{tabular}

du programme prégradué (MD) à la formation spécialisée (résidence), sans oublier l'éducation médicale continue. Il s'agit-là d'une recommandation préconisée dans le cadre du volet postdoctoral du projet sur l'AEMC.

L'élément de la norme proposée par le CAFMC pour assurer la responsabilité sociale serait placé au tout début du document. La première norme porte sur le contexte de «mission, planification, organisation et intégrité » :

Une faculté de médecine établit par écrit un énoncé de mission et des objectifs relatifs au programme d'éducation médicale, effectue une planification continue et s'est dotée de règlements administratifs écrits qui décrivent une structure organisationnelle efficace et des processus de gouvernance. Dans l'exercice de toutes ses activités internes et externes, la faculté de médecine fait preuve d'intégrité en se conformant de façon continue et documentée à des processus, politiques et pratiques équitables, impartiaux et efficaces.

L'élément 1.1 relatif à cette norme se lit comme suit :

Une faculté de médecine s'engage dans des processus de planification sur une base permanente et d'amélioration continue de la qualité, qui lui permettent de se fixer des objectifs programmatiques à court et à long terme, d'obtenir des résultats mesurables qu'elle utilise pour améliorer la qualité de ses programmes, et d'assurer un suivi efficace de la conformité du programme d'éducation médicale aux normes d'agrément.

Ensuite, plus précisément, sous la rubrique « responsabilité sociale » (1.1.1):

Une faculté de médecine s'engage à répondre aux préoccupations prioritaires en matière de santé des populations qu'elle est responsable de servir. La responsabilité sociale de la faculté de médecine : a) est exprimée dans son énoncé de mission ; b) est concrétisée dans le cadre de son programme éducatif par le biais des admissions, du contenu des programmes d'études, des types et des lieux où se déroulent les expériences éducatives et c) se traduit par des mesures de résultats précises.

Dans le cadre de la norme 6, l'élément 6.6 sur l'apprentissage par le service, stipule ce qui suit :

Le corps professoral d'une faculté de médecine veille à ce que le programme d'éducation médicale encourage et appuie la participation des étudiants en médecine à des activités d'apprentissage par le service communautaire et procure suffisamment d'occasions pour ces activités.

Ces normes demandent aux facultés de se pencher sérieusement sur la question et d'afficher des résultats sur plusieurs aspects de leur responsabilité sociale. 


\section{Discussion}

Le Canada a déterminé sa vision quant à l'avenir de l'éducation médicale. Cette vision s'articule autour de la responsabilité sociale des facultés de médecine. Ce thème est cher à nos doyens, au point qu'ils ont accepté d'être assujettis aux exigences très rigoureuses des normes d'accréditation.

D'autres pays pourront bénéficier des ressources et de l'expertise du Canada dans ces domaines. L'AFMC serait très fière de collaborer avec nos collègues de la francophonie pour faire avancer la question de la responsabilité sociale et de l'accréditation dans leur pays ou région.

\section{Déclaration d'intérêts}

L'auteur ne déclare de conflit d'intérêt en lien avec le contenu de cet article

\section{Approbation éthique}

Sans objet, non sollicitée

\section{Références}

1. Santé Canada. Imputabilité sociale - Une vision pour les facultés de médecine du Canada. 2001 [On-line]
Disponible sur : https://www.afmc.ca/pdf/pdf_sa_ vision_canadian_medical_schools_fr.pdf

2. L'Association des facultés de médecine du Canada. L'Avenir de l'éducation médicale au Canada: Une vision collective pour les études médicales prédoctorales. 2010 [On-line] Disponible sur: https://www.afmc.ca/future-of-medical-educationin-canada/medical-doctor-project/pdf/ vision_collective.pdf

3. L'Association des facultés de médecine du Canada, le Collège des médecins de famille du Canada, le Collège des médecins du Québec, le Collège royal des médecins et chirurgiens du Canada. L'Avenir de l'éducation médicale au Canada - Une vision collective pour les études médicales postdoctorales au Canada. 2012 [On-line] Disponible sur : https://www.afmc.ca/future-of-medical-education-in-canada/postgraduate-project/pdf/ FMEC_PG_Final-Report_FR.pdf

4. Comité d'agrément des facultés de médecine du Canada. Normes et éléments du CAFMC - Normes d'agrément des programmes d'éducation médicale en vue de l'obtention d'un diplôme en médecine (MD). 2014 [On-line] Disponible sur: https:// www.afmc.ca/sites/default/files/documents/en/ Accreditation/Normes_\%C3\%891\%C3\%A9ments CAFMC_AY_2016-17_\%28ao\%C3\%BBt_2015\%29.pdf

Correspondance et offprints : Geneviève Moineau, Association des facultés de médecine du Canada, 2733 rue Lancaster Road, suite/bureau 100, Ottawa, ON K1B 0A9, Canada Mailto : gmoineau@afmc.ca 